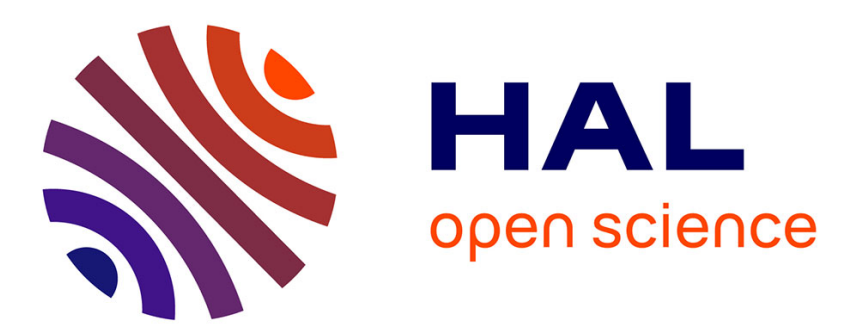

\title{
Translation Validation for Clock Transformations in a Synchronous Compiler
}

\author{
van Chan Ngo, Jean-Pierre Talpin, Thierry Gautier, Paul Le Guernic
}

\section{To cite this version:}

van Chan Ngo, Jean-Pierre Talpin, Thierry Gautier, Paul Le Guernic. Translation Validation for Clock Transformations in a Synchronous Compiler. FASE - ETAPS 2015, Apr 2015, London, United Kingdom. hal-01087795v3

\section{HAL Id: hal-01087795 \\ https://hal.inria.fr/hal-01087795v3}

Submitted on 17 Apr 2015

HAL is a multi-disciplinary open access archive for the deposit and dissemination of scientific research documents, whether they are published or not. The documents may come from teaching and research institutions in France or abroad, or from public or private research centers.
L'archive ouverte pluridisciplinaire HAL, est destinée au dépôt et à la diffusion de documents scientifiques de niveau recherche, publiés ou non, émanant des établissements d'enseignement et de recherche français ou étrangers, des laboratoires publics ou privés. 


\title{
Translation Validation for Clock Transformations in a Synchronous Compiler
}

\author{
Van Chan Ngo, Jean-Pierre Talpin, Thierry Gautier, and Paul Le Guernic \\ INRIA Rennes - Bretagne Atlantique, 35042 Rennes Cedex, France \\ \{firstname. lastname\} @inria.fr
}

\begin{abstract}
Translation validation was introduced as a technique to formally verify the correctness of code generators that attempts to ensure that program transformations preserve the semantics of input program. In this work, we adopt this approach to construct a validator that formally verifies the preservation of clock semantics during the SIGNAL compiler transformations. The clock semantics is represented as a first-order logic formula called clock model. We then introduce a refinement which expresses the preservation of clock semantics, as a relation on clock models. Our validator does not require any instrumentation or modification of the compiler, nor any rewriting of the source program.
\end{abstract}

Keywords: Formal Verification, Translation Validation, Certified Compiler, Smт Solver, Synchronous Data-Flow Languages.

\section{Introduction}

Motivation. Synchronous programming languages such as Signal, Lustre and ESTEREL propose a formal semantic framework to give a high-level specification of safety-critical software in automotive and avionics systems [10,13,2]. As other programming languages, synchronous languages are associated with a compiler. The compiler takes a source program, analyses and transforms it, performs optimizations, and finally generates executable code for a particular hardware platform or in some general-purpose programming languages. However, a compiler is a large and very complex program which often consists of hundreds of thousands, if not millions, lines of code, divided into multiple sub-systems and modules. The compilation process involves many analyzes, program transformations and optimizations. Some transformations and optimizations may introduce additional information, or constrain the compiled program. They may refine its meaning and specialize its behavior to meet a specific safety or optimization goal. Consequently, it is not uncommon that compilers silently issue an incorrect result in some unexpected context or inappropriate optimization goal. To circumvent compiler bugs, one can entirely rewrite the compiler with a theorem proving tool such as CoQ [8], or check that it is compliant to the DO-178C documents [22]. Nonetheless, these solutions yield a situation where any change of the compiler (e.g., further optimization and update) means redoing the proof. 
Another approach, which provides ideal separation between the tool under verification and its checker, is trying to verify that the output and the input have the same semantics. In this aim, translation validation was introduced in the 90's by Pnueli et al. [20,21], as a technique to formally verify correctness of code generators. Translation validators can be used to ensure that program transformations do not introduce semantic discrepancies, or to help debugging the compiler implementation.

Contribution. We consider the Signal compiler, in the first two phases, The clock information and Boolean abstraction are computed. The next phase is static scheduling and the final phase is the executable code generation. Obviously, one can prove that the input program and its transformed program at the final phase have the same semantics. However, we believe that a better approach consists in separating the concerns and proving for each phase the preservation of different kinds of semantic properties. In the case of a synchronous compiler such as Signal, the preservation of the semantics can be decomposed into the preservation of clock semantics, data dependencies, and value-equivalence of variables [18].

This paper focuses on constructing a validator that proves the preservation of clock semantics in the first two phases of the Signal compiler. The clock semantics of the source program and its transformed counterpart are formally represented as clock models. A clock model is a first-order logic formula with uninterpreted functions. This formula deterministically characterizes the presence/absence status of all discrete data-flows (input, output and local variables of a program) manipulated by the specification at a given logic instant. Given two clock models, a correct transformation relation between them is checked by the existence of their refinement relation, which expresses the semantic preservation of clock semantics. In the implementation, we apply our translation validation to the first two transformation steps of the compiler.

The remainder of this paper is organized as follows. Section 2 introduces the SignAl language. Section 3 presents the abstraction that represents the clock semantics in terms of first-order logic formula. In Section 4, we consider the definition of correct transformation on clock models which formally proves the conformance between the original specification and its transformed counterpart. The application of the verification process to the Signal compiler, and its integration in the Polychrony toolset [19] is addressed in Section 5. Section 6 presents related works and concludes our work.

\section{The SIGNAL Language}

SignAL $[5,11]$ is a polychronous data-flow language that allows the specification of multi-clocked systems, called polychrony models. Signal handles unbounded sequences of typed values $x(t)_{t \in \mathbb{N}}$, called signals, denoted as $x$. Each signal is implicitly indexed by a logical clock indicating the set of instants at which the signal is present, noted $C_{x}$. At a given instant, a signal may be present where it holds a value, or absent where it holds no value, denoted by $\perp$. Given two 
signals, they are synchronous iff they have the same clock. A process, written $\mathrm{P}$ or $\mathrm{Q}$, consists of the synchronous composition of equations over signals $x, y, z$, written $x:=y$ op $z$ or $x:=\mathrm{op}(y, z)$, where op is an operator. In particular, a process can be used as a basic pattern, by means of an interface that describes its parameters and its input and output signals. Moreover, a process can use other subprocesses, or even other processes as external parameters. A program is a process and the language is modular.

Data Domains. Data types consist of usual scalar types (Boolean, integer, float, complex, and character), enumerated types, array types, tuple types, and the special type event. It is a subtype of the Boolean which has only one value, true.

Operators. The core language consists of two kinds of "statement" defined by the following primitive operators: four operators on signals and two operators on processes. The operators on signals define basic processes with implicit clock relations while the operators on processes are used to construct complex processes with the parallel composition operator. In the delay operator, inf and sup denote the greatest lower bound and the least upper bound.

- Stepwise Functions: $y:=f\left(x_{1}, \ldots, x_{n}\right)$, where $f$ is a $n$-ary function on values, defines a basic process whose output $y$ is synchronous with $x_{1}, \ldots, x_{n}\left(C_{y}=\right.$ $\left.C_{x_{1}}=\ldots=C_{x_{n}}\right)$ and $\forall t \in C_{y}, y(t)=f\left(x_{1}(t), \ldots, x_{n}(t)\right)$.

- Delay: $y:=x \$ 1$ init $a$ defines a basic process such that $y$ and $x$ are synchronous $\left(C_{y}=C_{x}\right), y\left(t_{0}\right)=a$, and $\forall t \in C_{y} \wedge t>t_{0}, y(t)=x\left(t^{-}\right)$with $t_{0}=$ $\inf \left\{t^{\prime} \mid x\left(t^{\prime}\right) \neq \perp\right\}, t^{-}=\sup \left\{t^{\prime} \mid t^{\prime}<t \wedge x\left(t^{\prime}\right) \neq \perp\right\}$.

- Merge: $y:=x$ default $z$ defines a basic process which specifies that $y$ is present iff $x$ or $z$ is present $\left(C_{y}=C_{x} \cup C_{z}\right)$, and that $y(t)=x(t)$ if $t \in C_{x}$ and $y(t)=z(t)$ if $t \in C_{z} \backslash C_{x}$.

- Sampling: $y:=x$ when $b$ where $b$ is a Boolean signal, defines a basic process such that $\forall t \in C_{x} \cap C_{b} \wedge b(t)=$ true, $y(t)=x(t)$, and otherwise, $y$ is absent $\left(C_{y}=C_{x} \cap[b]\right.$, where $[b]=\left\{t \in C_{b} \mid b(t)=\right.$ true $\left.\}\right)$.

- Composition: If $\mathrm{P}_{1}$ and $\mathrm{P}_{2}$ are processes, then $\mathrm{P}_{1} \mid \mathrm{P}_{2}$, also denoted as $\left(\left|\mathrm{P}_{1}\right| \mathrm{P}_{2} \mid\right)$, is the process resulting of their parallel composition. This process consists of the composition of the systems of equations. The composition operator is commutative, associative, and idempotent.

- Restriction: $\mathrm{P}$ where $x$, where $\mathrm{P}$ is a process and $x$ is a signal, specifies a process by considering $x$ as local variable to $\mathrm{P}$ (i.e., $x$ is not accessible from outside $\mathrm{P}$ ).

Clock Relations. Clock relations can be defined explicitly: $y:=\hat{x}$ specifies that $y$ with event type is the clock of $x, C_{x}$. The synchronization $x \wedge y$ means that $x$ and $y$ have the same clock. The clock extraction from a Boolean signal is denoted by a unary when: when $b$. The clock union $x^{\wedge}+y$ defines a clock as the union $C_{x} \cup C_{y}$. In the same way, the clock intersection $x * y$ and the clock difference $x \wedge y$ define clocks $C_{x} \cap C_{y}$ and $C_{x} \backslash C_{y}$.

Example. The following SIGNAL program emits a sequence of values FB, FB $-1, \ldots$, 2, 1, from each value of a positive integer signal FB coming from its environment. We can see that the clock of the output signal is more frequent than that of the 
corresponds to logical conjunctions. Thus the clock model of $\mathrm{P}$ will be a conjunction $\Phi(\mathrm{P})=\bigwedge_{i=1}^{n} \phi\left(e q_{i}\right)$, whose atoms are $\widehat{x_{i}}, \widetilde{x_{i}}$, where $\phi\left(e q_{i}\right)$ is the abstraction of statement $e q_{i}$, and $n$ is the number of statements in the program. In the following, we present the abstraction corresponding to each SIGNAL operator.

Stepwise Functions. The functions which apply on signal values in the stepwise functions are usual logic operators (not, and, or), numerical comparison functions $(<,>,=,<=,>=, /=)$, and numerical operators $(+,-, *, /)$. In our experience working with the SIGNAL compiler, it performs very few arithmetical optimizations and leaves most of the arithmetical expressions intact. Every definition of a signal is determined explicitly by the input and register signals, otherwise program can not be compiled. This suggests that most of the implications will hold independently of the features of the numerical comparison functions and numerical operators and we can replace these operations by uninterpreted functions. By following the encoding procedure of [1], for every numerical comparison function and numerical operator (denoted by $\square$ ) occurring in an equation, we perform the following rewriting: i) Replace each $x \square y$ by a new variable $v_{\square}^{i}$ of the same type as the return value by $\square$. Two stepwise functions $x \square y$ and $x^{\prime} \square y^{\prime}$ are replaced by the same variable $v_{\square}^{i}$ iff $x, y$ are identical to $x^{\prime}$ and $y^{\prime}$, respectively; ii) For every pair of newly added variables $v_{\square}^{i}$ and $v_{\square}^{j}$, $i \neq j$, corresponding to the non-identical occurrences $x \square y$ and $x^{\prime} \square y^{\prime}$, add the implication $\left(\widetilde{x}=\widetilde{x^{\prime}} \wedge \widetilde{y}=\widetilde{y^{\prime}}\right) \Rightarrow \widetilde{v_{\square}^{i}}=\widetilde{v_{\square}^{j}}$ into the abstraction $\Phi(\mathrm{P})$. The abstraction $\phi\left(y:=f\left(x_{1}, \ldots, x_{n}\right)\right)$ of stepwise functions is defined by induction as follows: $\phi($ true $)=$ true and $\phi($ false $)=$ false $\phi(y:=x)=(\hat{y} \Leftrightarrow \hat{x}) \wedge(\hat{y} \Rightarrow(\widetilde{y}=\widetilde{x}))$; $\phi(y:=x)=(\hat{y} \Leftrightarrow \hat{x}) \wedge(\hat{y} \Rightarrow(\widetilde{y}=\widetilde{x})) \wedge(\hat{x} \Rightarrow \widetilde{x})$ if $x$ is an event signal; $\phi(y:=$ $\operatorname{not} x)=(\hat{y} \Leftrightarrow \hat{x}) \wedge(\hat{y} \Rightarrow(\widetilde{y} \Leftrightarrow \neg \widetilde{x})) ; \phi\left(y:=x_{1}\right.$ and $\left.x_{2}\right)=\left(\hat{y} \Leftrightarrow \widehat{x_{1}} \Leftrightarrow \widehat{x_{2}}\right) \wedge(\hat{y} \Rightarrow$ $\left.\left(\widetilde{y} \Leftrightarrow \widetilde{x_{1}} \wedge \widetilde{x_{2}}\right)\right) ; \phi\left(y:=x_{1}\right.$ or $\left.x_{2}\right)=\left(\hat{y} \Leftrightarrow \widehat{x_{1}} \Leftrightarrow \widehat{x_{2}}\right) \wedge\left(\hat{y} \Rightarrow\left(\widetilde{y} \Leftrightarrow \widetilde{x_{1}} \vee \widetilde{x_{2}}\right)\right)$; $\phi\left(y:=x_{1} \square x_{2}\right)=\left(\hat{y} \Leftrightarrow \widehat{v_{\square}^{i}} \Leftrightarrow \widehat{x_{1}} \Leftrightarrow \widehat{x_{2}}\right) \wedge\left(\hat{y} \Rightarrow\left(\widetilde{y}=\widetilde{v_{\square}^{i}}\right)\right)$.

Delay. Considering the delay operator, $y:=x \$ 1$ init $a$, its encoding $\phi(y:=$ $x \$ 1$ init $a)$ contributes to $\Phi(\mathrm{P})$ with the following conjunct: $(\hat{y} \Leftrightarrow \hat{x}) \wedge(\hat{y} \Rightarrow$ $\left.\left((\widetilde{y}=m \cdot x) \wedge\left(m \cdot x^{\prime}=\widetilde{x}\right)\right)\right) \wedge\left(m \cdot x_{0}=a\right)$. This encoding requires that at any instant, signals $x$ and $y$ have the same status (present or absent). To encode the value of the output signal as well, we introduce a memorization variable $m . x$ that stores the last value of $x$. The next value of $m . x$ is $m . x^{\prime}$ and it is initialized to $a$ in $m . x_{0}$.

Merge. The encoding of the merge operator, $y:=x$ default $z$, contributes to $\Phi(\mathrm{P})$ with the following conjunct: $(\hat{y} \Leftrightarrow(\hat{x} \vee \hat{z})) \wedge \hat{y} \Rightarrow((\hat{x} \wedge(\widetilde{y}=\widetilde{x})) \vee(\neg \hat{x} \wedge(\widetilde{y}=$ $\widetilde{z})))$

Sampling. The encoding of the sampling operator, $y:=x$ when $b$, contributes to $\Phi(\mathrm{P})$ with the following conjunct: $(\hat{y} \Leftrightarrow(\hat{x} \wedge \hat{b} \wedge \bar{b})) \wedge(\hat{y} \Rightarrow(\widetilde{y}=\widetilde{x}))$

Composition. Consider the composition of two processes $\mathrm{P}_{1}$ and $\mathrm{P}_{2}$. Its abstraction $\phi\left(\mathrm{P}_{1} \mid \mathrm{P}_{2}\right)$ is defined as follows: $\phi\left(\mathrm{P}_{1}\right) \wedge \phi\left(\mathrm{P}_{2}\right)$

Clock Relations. Given the above rules, we can obtain the following abstraction for derived operators on clocks. Here, $z$ is a signal of type event: $\phi(z:=\hat{x})=$ $(\hat{z} \Leftrightarrow \hat{x}) \wedge(\hat{z} \Rightarrow \bar{z}) ; \phi\left(x^{\wedge}=y\right)=\hat{x} \Leftrightarrow \hat{y} ; \phi\left(z:=x^{\wedge}+y\right)=(\hat{z} \Leftrightarrow(\hat{x} \vee \hat{y})) \wedge(\hat{z} \Rightarrow \bar{z}) ;$ 
$\phi(z:=x \hat{*} y)=(\hat{z} \Leftrightarrow(\hat{x} \wedge \hat{y})) \wedge(\hat{z} \Rightarrow \bar{z}) ; \phi\left(z:=x^{\wedge}-y\right)=(\hat{z} \Leftrightarrow(\hat{x} \wedge \neg \hat{y})) \wedge(\hat{z} \Rightarrow \bar{z}) ;$ $\phi(z:=$ when $b)=(\hat{z} \Leftrightarrow(\hat{b} \wedge \bar{b})) \wedge(\hat{z} \Rightarrow \bar{z})$.

Example. Applying the abstraction rules above, the clock semantics of the program DEC is represented by the following formula $\Phi(\mathrm{DEC})$, where $\mathrm{ZN}<=1$ and $\mathrm{ZN}-1$ are replaced by two fresh variables ZN1 and ZN2, and encoded by two uninterpreted function symbols $\mathrm{v}_{<=}^{1}$ and $\mathrm{v}_{-}^{1}$, respectively.

$$
\begin{aligned}
& (\widehat{F B} \Leftrightarrow \widehat{Z N 1} \wedge \widehat{Z N 1}) \wedge\left(\widehat{Z N 1} \Leftrightarrow \widehat{v_{<}^{1}} \Leftrightarrow \widehat{Z N}\right) \wedge\left(\widehat{Z N 1} \Rightarrow\left(\widehat{Z N 1}=\widehat{v_{<}^{1}}\right)\right) \\
\wedge & (\widehat{Z N} \Leftrightarrow \widehat{N}) \wedge\left(\widehat{Z N} \Rightarrow\left(\widehat{Z N}=m \cdot N \wedge m \cdot N^{\prime}=\tilde{N}\right)\right) \wedge\left(m \cdot N_{0}=1\right) \\
\wedge & (\widehat{N} \Leftrightarrow \widehat{F B} \vee \widehat{Z N 2}) \wedge(\widehat{N} \Rightarrow((\widehat{F B} \wedge \tilde{N}=\widehat{F B}) \vee(\neg \widehat{F B} \wedge \tilde{N}=\widehat{Z N 2}))) \\
\wedge & \left(\widehat{Z N 2} \Leftrightarrow \widehat{v_{-}^{1}} \Leftrightarrow \widehat{Z N}\right) \wedge\left(\widehat{Z N 2} \Rightarrow\left(\widehat{Z N 2}=\widetilde{v_{-}^{1}}\right)\right)
\end{aligned}
$$

In the following sections, we denote input, output, register, memorization and local variables used in a clock model by $I_{c l k}, O_{c l k}, R_{c l k}, M_{c l k}$ and $L_{c l k}$, respectively. Note that the memorization variables are introduced only by the translation into clock models, they are not original in the SIGNAL programs.

Definition 1 (Clock configuration). Consider a clock model $\Phi(\mathrm{P})$ over the set of variabels $\hat{X}$. A clock configuration $\widehat{I}$ is an interpretation over $\hat{X}$ such that it is a model of the first-order logic formula $\Phi(\mathrm{P})$.

For instance, $(\widehat{\mathrm{FB}} \mapsto$ true, $\widehat{\mathrm{N}} \mapsto$ true, $\widehat{\mathrm{ZN}} \mapsto$ true, $\widetilde{\mathrm{FB}} \mapsto 6, \tilde{\mathrm{N}} \mapsto 6, \widetilde{\mathrm{ZN}} \mapsto 1)$ is a clock configuration of $\Phi(\mathrm{DEC})$.

\subsection{Concrete Clock Semantics}

We rely on the basic elements of trace semantics [14] to define the clock semantics of a synchronous program. For each $x_{i} \in X$, we use $\mathbb{D}_{x_{i}}$ to denote its domain of values, and $\mathbb{D}_{x_{i}}^{\perp}=\mathbb{D}_{x_{i}} \cup\{\perp\}$ to denote its domain of values with absent value, where $\perp \notin \mathbb{D}_{x_{i}}$ denotes the absent value. Then, the domain of values of $X$ with absent value is defined as $\mathbb{D}_{X}^{\perp}=\bigcup_{i=1}^{n} \mathbb{D}_{x_{i}} \cup\{\perp\}$

Definition 2 (Clock events, clock traces). Given a non-empty set $X$, the set of clock events on $X$, denoted by $\mathcal{E} c_{X}$, is the set of all possible interpretations $I$ over $X$. The set of clock traces on $X$, denoted by $\mathcal{T} c_{X}$, is defined by the set of functions $T_{c}$ defined from the set $\mathbb{N}$ of natural numbers to $\mathcal{E} c_{X}$, denoted by $T_{c}: \mathbb{N} \longrightarrow \mathcal{E} c_{X}$

An interpretation $I$ is an assignment of values from $X$ to $\mathbb{D}_{X}^{\perp}$. The assignment $I(x)=\perp$ means $x$ holds no value while $I(x)=v$ means that $x$ holds the value $v$. The natural numbers represent the instants, $t=0,1,2, \ldots$, a trace $T_{c}$ is a chain of clock events. We denote the interpreted value of a variable $x_{i}$ at instant $t$ by $T_{c}(t)\left(x_{i}\right)$.

Definition 3 (Restriction clock trace). Given a non-empty set $X$, a subset $X_{1} \subseteq X$, and a clock trace $T_{c}$ being defined on $X$, the restriction of $T_{c}$ onto $X_{1}$ is denoted by $X_{1} \cdot T_{c}$. It is defined as $X_{1} \cdot T_{c}: \mathbb{N} \longrightarrow \mathcal{E} c_{X_{1}}$ such that $\forall t \in \mathbb{N}, \forall x \in$ $X_{1}, X_{1} \cdot T_{c}(t)(x)=T_{c}(t)(x)$. 
Let $X$ be the set of all signals in program $\mathrm{P}$. We write $[[\mathrm{P}]]_{c}$ to denote the clock semantics of $\mathrm{P}$ which is defined as the set of all possible clock traces on $X$. For any subset $X_{1} \subseteq X$, the set of all restriction clock traces on $X_{1}$ defines the clock semantics of $\mathrm{P}$ on $X_{1}$, denoted by $\left([[\mathrm{P}]]_{c}\right) \backslash X_{1}$.

Let $\Phi(\mathrm{P})$ be the clock model of the program P. We now define the concrete clock semantics of a clock model based on the notion of clock configurations. Given a clock configuration $\hat{I}$, the set of clock events according to $\hat{I}$ is the set of interpretations $I$ such that for every signal $x_{i}$, if $x_{i}$ holds a value then $\widehat{x_{i}}$ has the value true $\left(x_{i}\right.$ is present), and $\widetilde{x_{i}}$ holds the same value as $x_{i}$. Otherwise, $\widehat{x_{i}}$ has the value false (meaning $x_{i}$ is absent). The set of clock events according to $\hat{I}$ and the set of all clock events of $\Phi(\mathrm{P})$ are computed as follows:

$$
\begin{aligned}
S_{\mathcal{E}_{c_{X}}}(\hat{I})= & \left\{I \in \mathcal{E}_{c_{X}} \mid \forall x_{i} \in X,\left(I\left(x_{i}\right)=\hat{I}\left(\widetilde{x}_{i}\right) \wedge \hat{I}\left(\widehat{x_{i}}\right)=\text { true }\right)\right. \\
& \left.\vee\left(I\left(x_{i}\right)=\perp \wedge \hat{I}\left(\widehat{x_{i}}\right)=\text { false }\right)\right\} \\
S_{\mathcal{E}_{c_{X}}}(\Phi(\mathrm{P}))= & \bigcup_{\hat{I}=\Phi(\mathrm{P})} S_{\mathcal{E}_{c_{X}}(\hat{I})}
\end{aligned}
$$

With a set of clock events $S_{\mathcal{E}_{c_{X}}}(\Phi(\mathrm{P}))$, the concrete clock semantics of $\Phi(\mathrm{P})$ is defined by the following set of clock traces $\Gamma(\Phi(\mathrm{P}))=\left\{T_{c} \in \mathcal{T} c_{X} \mid \forall t, T_{c}(t) \in\right.$ $\left.S_{\mathcal{E}_{c_{X}}}(\Phi(\mathrm{P}))\right\}$. For any subset $X_{1} \subseteq X$, the concrete clock semantics of $\Phi(\mathrm{P})$ on $X_{1}$ is defined as $\Gamma(\Phi(\mathrm{P}))_{\backslash X_{1}}=\left\{X_{1} \cdot T_{c} \mid T_{c} \in \mathcal{T} c_{X}\right.$ and $\left.\forall t, T_{c}(t) \in S_{\mathcal{E}_{c_{X}}}(\Phi(\mathrm{P}))\right\}$. Due to the lack of space, we do not present the proof of soundness of our abstraction.

\section{Clock Model Translation Validation}

We adopt the translation validation approach [20,21] to formally verify that the clock semantics is preserved for every transformation of the compiler. In order to apply the translation validation to the transformations, we capture the clock semantics of the original program and its transformed counterpart by means of clock models. Then we introduce a refinement relation which expresses the preservation of clock semantics, as relation on clock models.

\subsection{Clock Refinement}

Let $\Phi(\mathrm{A})$ and $\Phi(\mathrm{C})$ be two clock models of programs A and C, to which we refer respectively as a source program and its transformed counterpart produced by the compiler. We denote the sets of all signals in A, C by $X_{A}$ and $X_{C}$, respectively. The corresponding sets of variables which are used to construct the clock models are denoted by $\widehat{X_{A}}$ and $\widehat{X_{C}}$. Consider the finite set of common signals $X=$ $X_{A} \cap X_{C}$ and the set of common variables which are used to construct the clock models is $\widehat{X}=\widehat{X_{A}} \cap \widehat{X_{C}}$, we say that $\mathrm{A}$ and $\mathrm{C}$ have the same clock semantics on $X$ if $\Phi(\mathrm{A})$ and $\Phi(\mathrm{C})$ have the same set of concrete restriction clock traces on $X$ :

$$
\forall X . T_{c} \cdot\left(X . T_{c} \in \Gamma(\Phi(\mathrm{C}))_{\backslash X} \Leftrightarrow X . T_{c} \in \Gamma(\Phi(\mathrm{A}))_{\backslash X}\right)
$$

In fact, the compilation makes the transformed program more concrete. For instance, when the SignAL compiler performs the "endochronization" which is 
used to generate the sequential executable code, the signal with the fastest clock is always present in the generated code. Moreover, compilers perform transformations and optimizations for removing or eliminating some redundant behaviors of the source program (e.g., eliminating subexpressions, trivial clock relations). Consequently, the above requirement is too strong to be practical. Hence, we have to relax it as follows:

$$
\forall X . T_{c} .\left(X . T_{c} \in \Gamma(\Phi(\mathrm{C}))_{\backslash X} \Rightarrow X . T_{c} \in \Gamma(\Phi(\mathrm{A}))_{\backslash X}\right)
$$

It expresses that every restriction clock trace of $\Phi(\mathrm{C})$ is also a clock trace of $\Phi(\mathrm{A})$ on $X$, or $\Gamma(\Phi(\mathrm{C}))_{\backslash X} \subseteq \Gamma(\Phi(\mathrm{A}))_{\backslash X}$. We say that $\Phi(\mathrm{C})$ is a correct clock transformation of $\Phi(\mathrm{A})$, or $\Phi(\mathrm{C})$ is a clock refinement of $\Phi(\mathrm{A})$ on $X$, denoted by $\Phi(\mathrm{C}) \sqsubseteq_{c l k} \Phi(\mathrm{A})$.

Proposition 1. The clock refinement is reflexive and transitive

Proof. Proposition 1 is proved based on the clock refinement definition. $\Phi(\mathrm{P}) \sqsubseteq_{c l k}$ $\Phi(\mathrm{P})$ since $\Gamma(\Phi(\mathrm{P}))_{\backslash X} \subseteq \Gamma(\Phi(\mathrm{P}))_{\backslash X}$. For every clock trace $X . T_{c} \in \Gamma\left(\Phi\left(\mathrm{P}_{1}\right)\right)_{\backslash X}$, $\Phi\left(\mathrm{P}_{1}\right) \sqsubseteq_{c l k} \Phi\left(\mathrm{P}_{2}\right)$ on $X$ implies $X . T_{c} \in \Gamma\left(\Phi\left(\mathrm{P}_{2}\right)\right)_{\backslash X}$. Since $\Phi\left(\mathrm{P}_{2}\right) \sqsubseteq c l k \Phi\left(\mathrm{P}_{3}\right)$ on $X$, we have $X . T_{c} \in \Gamma\left(\Phi\left(\mathrm{P}_{3}\right)\right)_{\backslash X}$, or $\Phi\left(\mathrm{P}_{1}\right) \sqsubseteq_{c l k} \Phi\left(\mathrm{P}_{3}\right)$ on $X$.

\subsection{Adaptation to SIGNAL Compiler}

We will adapt the definition of the above general clock refinement to the case of the Signal compiler. We need to consider the following factors [4]. A first consideration is that the programs take the inputs from their environment and the register values. Then, they calculate the outputs to react with the environment. In general, the programs can use some local variables to make the output calculations. However, from the outside, the natural observation of the programs is the snapshot of the values of the input and output signals. In our context, it is the snapshot of the presence of the input and output signals. For example, for the program DEC, the observation is the tuple of the presence of the signals (FB, N) at a considered instant.

A second consideration is that in the compilation process of the SignaL compiler, the local signals in the source program do not necessarily have counterparts in the transformed program. However, all input and output signals are preserved in the transformations and are represented by identical names in the transformed program. Moreover, all signals in the $R$ set are also preserved in the transformations. Therefore, it is natural to choose the snapshot of the presence of the input and output signals to be the observation for the transformed program.

These considerations let us adapt the above definition of clock refinement as follows. Let $X_{A}$ and $X_{C}$ be the sets of all signals in the source program A and its counterpart transformed program C. We write $X_{I O}$ to denote the set of common input and output signals. We say that $\mathrm{C}$ is correct transformation of $\mathrm{A}$ if at any instant, the tuples of values representing the presence of the signals in $X_{I O}$ are the same in both programs. In other words, $\Phi(\mathrm{C}) \sqsubseteq_{c l k} \Phi(\mathrm{A})$ on $X_{I O}$. 


\subsection{Proving Clock Refinement by SMT}

Our aim is proving that $\Phi(\mathrm{C})$ refines $\Phi(\mathrm{A})$ on $X_{I O}$. Let $\widehat{X_{A}}, \widehat{X_{C}}$ and $\widehat{X_{I O}}$ be the set of variables which are used to construct $\Phi(\mathrm{A}), \Phi(\mathrm{C})$ and the set of common variables between the two clock models. For every variable in the clock model $\Phi(\mathrm{C})$ except the common variables in $\widehat{X_{I O}}$, we added "c" as superscript to distinguish them from the variables in the clock model of the input program. The standard way of proving the existence of the clock refinement is based on the following elements:

- The identification of a variable mapping that maps the non input/output variables from the clock model $\Phi(\mathrm{A})$ to the non input/output variables in the clock model $\Phi(\mathrm{C})$. We denote the mapping by: $\widehat{X_{A} \backslash} \widehat{X_{I O}}=\alpha\left(\widehat{X_{C}} \backslash \widehat{X_{I O}}\right)$

- The premises of a rule such that if the premises hold, then the conclusion, $\Phi(\mathrm{C})$ refines $\Phi(\mathrm{A})$, is true. The premise is presented in Fig. 1.

For a variable mapping $\widehat{X_{A}} \backslash \widehat{X_{I O}}=\alpha\left(\widehat{X_{C}} \backslash \widehat{X_{I O}}\right)$,
Premise $\forall \hat{I}$ over $\widehat{X_{A}} \cup \widehat{X_{C}} \cdot(\hat{I} \models \Phi(\mathrm{C}) \Rightarrow \hat{I} \models \Phi(\mathrm{A}))$

Conclusion $\Phi(\mathrm{C}) \sqsubseteq_{c l k} \Phi(A)$ on $X_{I O}$

Fig. 1: Rule CLKREF

The rule CLKREF indicates that for any interpretation $\hat{I}$ over $\widehat{X_{A}} \cup \widehat{X_{C}}$ such that the variable mapping is evaluated to true, $\hat{I}$ is a clock configuration of $\Phi(\mathrm{C})$ then it is also a clock model of $\Phi(\mathrm{A})$. Then there exists a clock refinement for $(\Phi(\mathrm{C}), \Phi(\mathrm{A}))$. The rule CLKREF is sound based on the following theorem.

Theorem 1. For a variable mapping $\widehat{X_{A}} \backslash \widehat{X_{I O}}=\alpha\left(\widehat{X_{C}} \backslash \widehat{X_{I O}}\right)$, if the formula $\Phi(\mathrm{C}) \Rightarrow \Phi(\mathrm{A})$ is valid, then $\Phi(\mathrm{C}) \sqsubseteq$ clk $\Phi(\mathrm{A})$ on $X_{I O}$.

Proof. To prove it, we have to show that for every interpretation $\hat{I}$ over $\hat{X}=$ $\widehat{X_{A}} \cup \widehat{X_{C}}$ such that it is evaluated to true. If $\hat{I} \models(\Phi(\mathrm{C}) \Rightarrow \Phi(\mathrm{A}))$, then $\Gamma(\Phi(\mathrm{C}))_{\backslash X_{I O}} \subseteq \Gamma(\Phi(\mathrm{A}))_{\backslash X_{I O}}$. Given $X_{I O} \cdot T_{c} \in \Gamma(\Phi(\mathrm{C}))_{\backslash X_{I O}}$, it means that $\forall t, T_{c}(t) \in S_{\mathcal{E}_{c_{X}}}(\Phi(\mathrm{C}))$. Since for every interpretation $\hat{I}, \hat{I}=\Phi(\mathrm{C})$ implies that $\hat{I}=\Phi(\mathrm{A}))$, thus $S_{\mathcal{E}_{c_{X}}}(\Phi(\mathrm{C})) \subseteq S_{\mathcal{E}_{c_{X}}}(\Phi(\mathrm{A}))$ under the variable mapping. We get $T_{c}(t) \in S_{\mathcal{E}_{c_{X}}}(\Phi(\mathrm{A}))$ for every $t$. Therefore, we have $T_{c} \in \Gamma(\Phi(\mathrm{A}))$.

Consider a variable $x \in \widehat{X_{A}} \backslash \widehat{X_{I O}}$, the mapping $\alpha_{x}$ of the variable mapping defines the value of $x$ in the clock model $\Phi(\mathrm{A}) \alpha$-related to the value represented by the clock model $\Phi(\mathrm{C})$. We therefore need to describe the mappings $\alpha_{x}$ for $x^{c} \in$ $\widehat{X_{C}} \backslash \widehat{X_{I O}}=M_{c l k} \cup R_{c l k} \cup L_{c l k}$. Recall that every register signal $s$, we introduce memorization variables m.s, m.s $s^{\prime}$ in the clock model $\Phi(\mathrm{A})$, and the corresponding memorization variables $m . s^{c}, m . s^{\prime c}$ in the clock model $\Phi(\mathrm{C})$. Therefore, we define the following instance of the $\alpha$ mapping for each register signal $s: \widetilde{s}=\widetilde{s^{c}} \Rightarrow m . s=$ $m . s^{c} \wedge m . s^{\prime}=m . s^{\prime c}$.

For example, the mapping for the variables m.N, m.N $N^{\prime}, m \cdot N^{c}$, and $m . N^{\prime c}$ will be given by the formula: $\widetilde{N}=\widetilde{N^{c}} \Rightarrow m \cdot N=m \cdot N^{c} \wedge m \cdot N^{\prime}=m \cdot N^{\prime c}$. 
It remains to define the instance of the mapping $\alpha$ for variables $\hat{l}, \tilde{l} \in R_{c l k} \cup$ $L_{c l k}$ in the clock model $\Phi(\mathrm{A})$ which correspond to the local or register signal named $l$ in the program. In a Signal program, one signal is defined by an equation $l=e q$, if we follow the definitions of all output and local signals in this equation and apply successively substitutions, then we get that the equation is constructed only by the input and register signals. This property is yielded since the SigNAL program is determinate, meaning that all definitions of signals are defined determinately by the input and register signals, and the compilers rejects all non-determinate program. Equivalently, in the corresponding clock model $\Phi(\mathrm{A})$, the output, register and local variables are determinately defined by the input $I$ and memorization $M$ variables. The definition is written in the clock model in the form $\hat{l} \Leftrightarrow \hat{f} \wedge(\hat{l} \Rightarrow \widetilde{l}=\widetilde{f})$ or $\hat{l} \Leftrightarrow \hat{f} \wedge(\hat{l} \Rightarrow \widetilde{l}=\widetilde{f}) \wedge \widetilde{f_{0}}$, where $\hat{f}, \widetilde{f}$ and $\widetilde{f}_{0}$ are the formulas which define the clock relation, the value, and the initial value of the signal $l$ in the clock model $\Phi(\mathrm{A})$. Therefore, we define the following instance of the $\alpha$ mapping in the clock model corresponding to each register or local signal $l: \hat{l} \Leftrightarrow \hat{f} \wedge(\hat{l} \Rightarrow \widetilde{l}=\widetilde{f})$ or $\hat{l} \Leftrightarrow \hat{f} \wedge(\hat{l} \Rightarrow \widetilde{l}=\widetilde{f}) \wedge \widetilde{f}$.

For example, the mapping for the variables $\widehat{Z N}$ and $\widetilde{Z N}$ in the clock model $\Phi(\mathrm{DEC})$ corresponding to the local variable $Z N$ in the program DEC will be given by the formula: $(\widehat{Z N} \Leftrightarrow \widehat{N}) \wedge\left(\widehat{Z N} \Rightarrow\left(\widetilde{Z N}=m \cdot N \wedge m . N^{\prime}=\widetilde{N}\right)\right) \wedge\left(m \cdot N_{0}=1\right)$.

Therefore, the variable mapping $\widehat{X_{A}} \backslash \widehat{X_{I O}}=\alpha\left(\widehat{X_{C}} \backslash \widehat{X_{I O}}\right)$ is expressed as the following formula:

$$
\begin{aligned}
& \bigwedge_{m \cdot s \in M}\left(\widetilde{s}=\widetilde{s}^{c} \Rightarrow m . s=m . s^{c}\right) \wedge \bigwedge_{\hat{l}, \widetilde{l} \in S \cup L}(\hat{l} \Leftrightarrow \hat{f} \wedge(\hat{l} \Rightarrow \widetilde{l}=\widetilde{f})) \text { or } \\
& \bigwedge_{m . s \in M}\left(\widetilde{s}=\widetilde{s^{c}} \Rightarrow m . s=m \cdot s^{c}\right) \wedge \bigwedge_{\hat{l}, \tilde{l} \in S \cup L}\left(\hat{l} \Leftrightarrow \hat{f} \wedge(\hat{l} \Rightarrow \widetilde{l}=\widetilde{f}) \wedge \widetilde{f}_{0}\right)
\end{aligned}
$$

To solve the validity of the formula $(\Phi(\mathrm{C}) \Rightarrow \Phi(\mathrm{A}))$ in Theorem 1 under the variable mapping, a SMT solver is needed since this formula involves non-Boolean variables and uninterpreted functions (using a SAT solver would not be sufficient). A SMT solver decides the satisfiability of arbitrary logic formulas of linear real and integer arithmetic, scalar types, other user-defined data structures, and uninterpreted functions. If the formula belongs to the decidable theory, the solver gives two types of answers: sat when the formula has a model (there exists an interpretation that satisfies it); or unsat, otherwise. In our case, we will ask the solver to check whether the formula $\neg\left(\Phi(\mathrm{C}) \wedge \widehat{X_{A}} \backslash \widehat{X_{I O}}=\alpha\left(\widehat{X_{C} \backslash \widehat{X_{I O}}}\right) \Rightarrow \Phi(\mathrm{A})\right)$ is unsatisfiable, since this formula is unsatisfiable iff $\models\left(\Phi(\mathrm{C}) \wedge \widehat{X_{A} \backslash \widehat{X_{I O}}}=\right.$ $\left.\alpha\left(\widehat{X_{C}} \backslash \widehat{X_{I O}}\right) \Rightarrow \Phi(\mathrm{A})\right)$. In our translation validation, the clock models which are constructed from Boolean or numerical variables and uninterpreted functions belong to a part of first-order logic which has a small model property according to [3]. The numerical variables are involved only in some implications with uninterpreted functions such as $\left(\widetilde{x}=\widetilde{x^{\prime}} \wedge \widetilde{y}=\widetilde{y^{\prime}}\right) \Rightarrow \widetilde{v_{\square}^{i}}=\widetilde{v_{\square}^{j}}$.

In addition, the formula is quantifier-free. This means that the check of satisfiability can be established by examining a certain finite cardinality of models. Therefore, the formula can be solved efficiently and significantly improves the scalability of the solver. 


\section{Implementation}

This section describes the implementation of our validator and some adaptation when the translation validation is applied to the real SignAL compiler. We also show the previously unknown bugs have been detected so far by our validator.

\subsection{Towards Certified Compiler}

Given a program $\mathrm{P}$, with an unverified compiler, the compilation process can be represented in the following pseudo-code, where $C p(\mathrm{P})$ is the compilation step from the source program $\mathrm{P}$ to either compiled code $I R(\mathrm{P})$ or compilation errors:

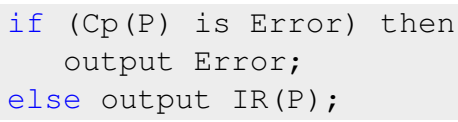

Now, the compilation is followed by our refinement verification which checks that the transformed program $I R(\mathrm{P})$ refines $\mathrm{P}$ w.r.t. the clock semantics:

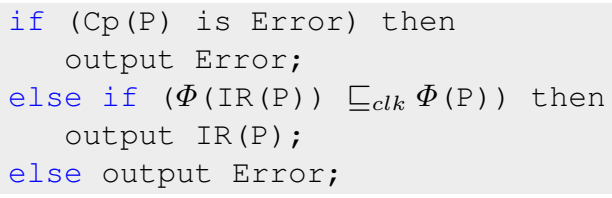

This will provide a formal guarantee as strong as that provided by a certified compiler in case the correctness of the validator is proved. We describe the main components of the implementation which is integrated in the existing POLYCHRONY toolset [19] to prove the preservation of clock semantics of the SIGNAL compiler. We are interested here in the first phase: clock calculation and Boolean abstraction where the intermediate forms of the source program of are expressed in the SignAL language itself.

At a high level, our validator, which is depicted in Figure 2, works as follows. First, it takes the input program P and its transformed counterpart P_BASIC_TRA, and constructs the corresponding clock models. These clock models are combined as the formula $\left(\Phi\left(\mathrm{P}_{\text {BASIC_TRA }}\right) \Rightarrow \Phi(\mathrm{P})\right)$. In the solving phase, it checks the validity of the formula $\Phi($ P_BASIC_TRA $) \Rightarrow \Phi(\mathrm{P})$. The result of this check can be exploited for the preservation of clock semantics of the transformations. If the formula is not valid then it emits a compiler bug. Otherwise, the compiler continues its work. The same procedure is applied for the other steps of the compiler. Finally, our verification process asserts that $\Phi($ P_BOOL_TRA $) \sqsubseteq c l k$ $\Phi\left(\mathrm{P} \_\right.$BASIC_TRA $) \sqsubseteq_{c l k} \Phi(\mathrm{P})$ along the transformations of the compiler. We delegate the checking of the clock refinement to a SMT solver. For our experiments, we consider the YICES [9] solver, which is one of the best solvers at the SMTComP competition [23].

\subsection{Detected Bugs}

So far, our validator has revealed three previously unknown bugs in the compilation of the Signal compiler. The first problem was introduced when multiple 


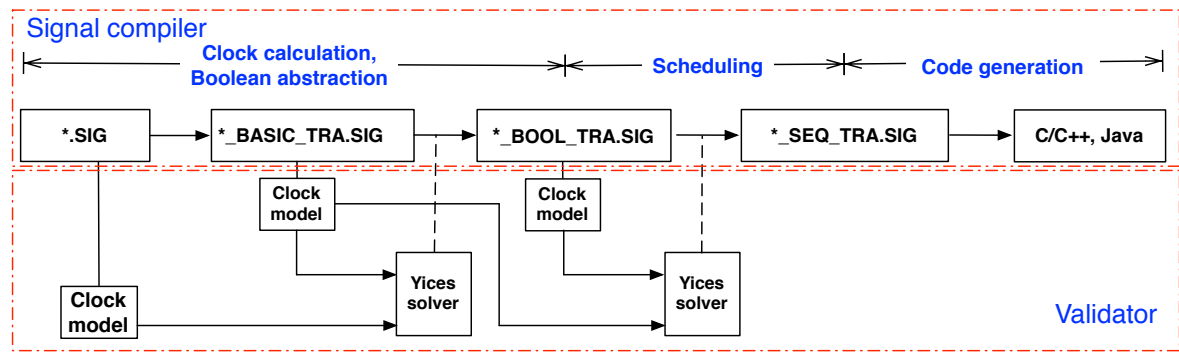

Fig. 2: The Integration within POLYCHRONY Toolset

constraints condition a clock such as in the following segment of SignaL program and its clock calculation part in transformed programs:

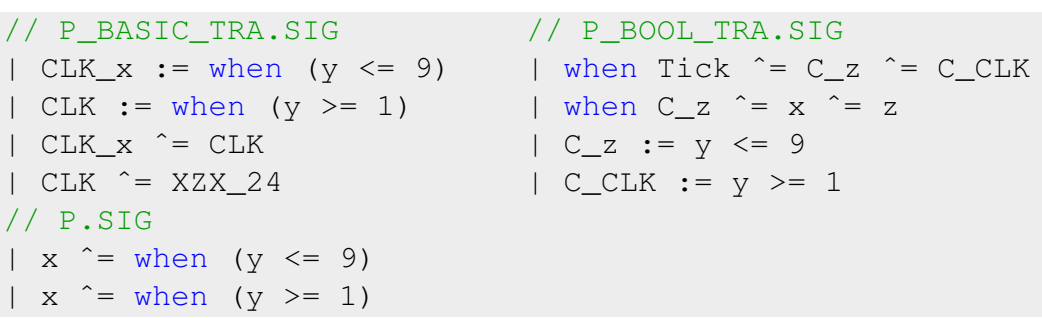

In the transformed counterpart P_BASIC_TRA, the introduction of signal XZX_24 and the synchronization between CLK and XZX_24 cause the incorrect specification of clocks, the signal $x$ might be absent when XZX_24 is absent, which is not the case in $\mathrm{P}$, nor in P_BOOL_TRA). This bug was caught by our validator when it found that $\Phi($ P_BOOL_TRA $) \nsubseteq_{c l k} \Phi($ P_BASIC_TRA $)$.

The second problem is the wrong implementation of xor operator as shown in the followng program. The validator detects this bug with the fact that $\Phi\left(\mathrm{P}_{-}\right.$BASIC_TRA $) \nsubseteq_{c l k} \Phi(\mathrm{P})$.

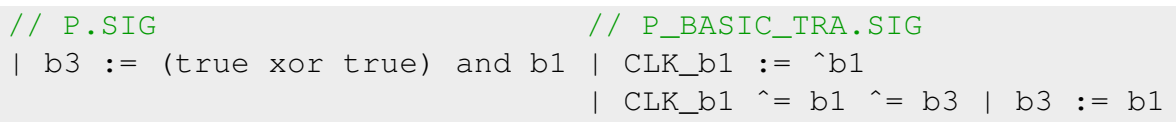

The last problem detected was not found by the translation validation but was indirectly discovered when trying to apply it. It occurred in a program in which a merge operator with a constant signal was used, such as $y:=$ 1 default $x$. In this case, the code generation phase of the compiler dealt wrongly with the clock context of a constant signal by introducing a syntax error in the generated $\mathrm{C}$ code. The bug and its fix are given by:

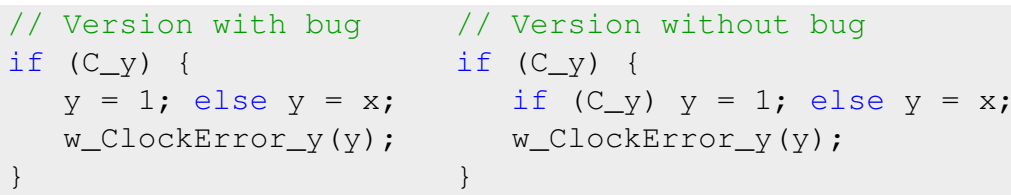




\section{Related Work and Conclusion}

The notion of translation validation was introduced in [20,21] by A. Pnueli et al. to verify the code generator of SigNAL. In that work, the authors define a language of symbolic models to represent both the source and target programs, called Synchronous Transition Systems (STS). A STs is a set of logic formulas which describes the functional and temporal constraints of the whole program and its generated $\mathrm{C}$ code. Then they use BDD [6] representations to implement the symbolic STS models, and their proof method uses a solver to reason on constraints over signals. The drawback of this approach is that it does not capture explicitly the clock semantics. Additionally, for a large program, the formula is very large, including numerical expressions that cause some inefficiency. Moreover, the whole calculation of a synchronous program or the corresponding generated code is considered as one atomic transition in STS, thus it does not capture the data dependencies between signals and does not explicitly prove the preservation of abstract clocks in the compiler transformations.

Another related work is the static analysis of SIGNAL programs for efficient code generation [12]. In a similar way as we do, the authors formalize the abstract clocks and clock relations as first-order logic formulas with the help of interval abstraction technique. The objective is to make the generated code more efficient by detecting and removing the dead-code segments (e.g., segment of code to compute a data-flow which is always absent). They determine the existence of empty clocks, mutual exclusion of two or more clocks, or clock inclusions, by reasoning on the formal model using a SMT solver.

Some other works have adopted the translation validation approach in verification of transformations, and optimizations. In [16], the translation validation is used to verify several common optimizations such as common subexpression elimination, register allocation, and loop inversion. The validator is simulationbased, that means it checks the existence of a simulation relation between two programs. Leroy et al. [15,7] used this technique to develop the COMPCERT highassurance $\mathrm{C}$ compiler. The programs before and after the transformations and optimizations of the compiler are represented in a common intermediate form, then the preservation of semantics is checked by using symbolic execution and the proof assistant CoQ. It also has shown that translation validation can be used to validate advanced loop optimizations such as software pipelining as in [25]. Tristan et al. [24] recently proposed a framework for translation validation of LLVM optimizer. For a function and its optimized counterpart, they compute a shared value-graph. The graph is normalized (roundly speaking, the graph is reduced). After the normalizing, if the outputs of two functions are represented by the same sub-graph, they can safely conclude that two functions are equivalent.

With the same purpose, in the work of [17], we encode the source SIGNAL programs and their transformations with Polynomial Dynamical Systems (PDS), and we prove that the transformations preserve the abstract clocks and clock relations of the source programs. This approach uses simulation relation in model checking techniques, and it suffers from the increasing of the state-space when it deals with large programs. On the contrary, in our present work, the abstract 
clocks and clock relations are described as a logic formula over Boolean variables. Thanks to the efficiency of SMT solver implementation in processing formulas over Boolean variables and uninterpreted functions, our approach can deal with large programs whose number of variables is large. This situation generally makes the state-space explosion problem in model checking techniques.

The present paper provides a proof of correctness of a the synchronous dataflow compiler. We have presented a technique based on SMT solving to prove the preservation of clock semantics during the compilation. Namely, we have shown that implicit clock relations, describing the discrete timing model of a dataflow specification, are preserved in their implementation. The desired behavior of a given source program and the transformed one are represented as clock models. A refinement relation between source and transformed programs is used to express the preservation, which is checked by using a SMT solver. We have constructed and integrated our validator within the POLYCHRONY toolset to prove the correctness of the Signal compiler.

We believe that our validator must have the following features to be effective and realistic. First, we do not modify or instrument the compiler, and we treat the compiler as a "black box". Hence the validator is not affected by some future update or modification of the compiler. We only need some additional information about the mapping between original names and potential new names of local variables. Our approach consists in applying formal methods to the compiler transformations themselves in order to automatically generate formal evidence that the clock semantics of the source program is preserved during program transformations, as per applicable qualification standard. Second, it is important that the validator can be scaled to large programs. For this purpose, we represent the desired program semantics using a scalable abstraction and we use efficient SmT libraries [9] to achieve the expected goals: traceability and formal evidence. Moreover, this approach provides an attractive alternative to develop a certified compiler for a synchronous language since in general the validator is much smaller and easier to verify than the compiler it validates.

\section{References}

1. W. Ackerman, Solvable Cases of the Decision Problem. Study in Logic and the Foundations of Mathematics. North-Holland, Amsterdam, 1954.

2. G. Berry, The Foundations of Esterel. In Proof, Language and Interaction: Essay in Honor of Robin Milner, MIT Press, 2000.

3. E. Borger, E. Gradel, and Y. Gurevich, The Classical Decision Problem. SpingerVerlag, 1996.

4. L. Besnard, T. Gautier, P. Le Guernic, and J-P. Talpin, Compilation of Polychronous Data Flow Equations. In Synthesis of Embedded Software, Springer, 2010.

5. A. Benveniste and P. LeGuernic, Hybrid Dynamical Systems Theory and the Signal Language. IEEE Transactions on Automatic Control. 35(5):535-546, May 1990.

6. R. Bryant, Graph-based Algorithms for Boolean Function Manipulation. IEEE Transactions on Computers, C-35(8):677-691, Aug 1986.

7. Inria, The CompCert Project. http://compcert.inria.fr. 
8. Inria, The Coq Proof Assitant. http://coq.inria.fr.

9. B. Dutertre, and L. de Moura, Yices Sat-solver. http://yices.csl.ri.com, 2009.

10. A. Gamatié, Designing Embedded Systems with the Signal Programming Language: Synchronous, Reactive Specification. Springer, New York. ISBN 978-1-4419-0940-4, 2009.

11. T. Gautier and P. Le Guernic and L. Besnard, Signal, a Declarative Language for Synchronous Programming of Real-time Systems. Proc. 3rd. Conf. on Functional Programming Languages and Computer Architecture, LNCS 274, May 1990.

12. A. Gamatié, and L. Gonnord, Static Analysis of Synchronous Programs in Signal for Efficient Design of Multi-Clocked Embedded Systems. In ACM SIGPLAN/SIGBED Conference on Languages, Compilers, Tools and Theory for Embedded Systems - LCTES'2011. Chicago, IL, USA, April 2011.

13. N. Halbwachs, A Synchronous Language at Work: the Story of Lustre. In 3th ACM-IEEE International Conference on Formal Methods and Models for Codesign (MEMOCODE'05), Jul 2005.

14. P. Le Guernic, and T. Gautier, Advanced Topics in Data-flow Computing, Chapter Data-flow to von Neumann: the Signal Approach. Prentice-Hall. pp.413-438, 1991.

15. X. Leroy, Formal Certification of a Compiler Back-end, or Programming a Compiler with a Proof Assistant. In 33rd Symposium Principles of Programming Languages, pp.42-54. ACM Press, 2006.

16. G.C. Necula, Translation Validation for an Optimizing Compiler. In Proceeding PLDI'00 Proceedings of the ACM SIGPLAN 2000 Conference on Programming Language Design and Implementation. pp.83-94, May 2000.

17. V. C. Ngo, J-P. Talpin, T. Gautier, P. Le Guernic, and L. Besnard, Formal Verification of Compiler Transformations on Polychronous Equations. In Proceedings of IFM'12, LNCS 7321. pp.113-127, 2012.

18. V. C. Ngo. Formal Verification of a Synchronous Data-flow Compiler: from Signal to $C$. In $\mathrm{PhD}$ thesis, 2014.

19. Inria/Espresso, Polychrony Toolset. http://www.irisa.fr/espresso/Polychrony.

20. A. Pnueli, M. Siegel, and E. Singerman, Translation Validation. In B. Steffen, editor, 4th Intl. Conf. TACAS'98. LNCS 1384. pp.151-166, 1998.

21. A. Pnueli, O. Shtrichman, and M. Siegel, Translation Validation: From Signal to C. In Correct Sytem Design Recent Insights and Advances. LNCS 1710. pp.231-255, 2000 .

22. RTCA, DO-178C. http://rtca.org

23. A. Stump, and M. Deters, SMT-Comp. http://www.smtcomp.org/2009, 2009.

24. J-B. Tristan, P. Govereau, and G. Morrisett, Evaluating Value-graph Translation Validation for LLVM. In ACM SIGPLAN Conference on Programming and Language Design Implementation. California, June 2011.

25. J-B. Tristan, and X. Leroy, A Simple, Verified Validator for Software Pipelining. In 37th Principles of Programming Languages, pp.83-92. ACM Press, 2010. 